\title{
Predictors of Ischemic Stroke After Hip Operation: A Population-Based Study
}

Alina S. Popa, Mo ${ }^{1}$ Alejandro A. Rabinstein, $\mathrm{MD}^{2}$ Paul M. Huddleston, $\mathrm{MD}^{3}$ Dirk R. Larson, $\mathrm{ms}^{4}$ Rachel E. Gullerud, Bs ${ }^{4}$ Jeanne M. Huddleston, MD ${ }^{1}$

\author{
${ }^{1}$ Division of Hospital Internal Medicine, Mayo Clinic, Rochester, Minnesota. \\ ${ }^{2}$ Department of Neurology, Mayo Clinic, Rochester, Minnesota. \\ ${ }^{3}$ Department of Orthopedic Surgery, Mayo Clinic, Rochester, Minnesota. \\ ${ }^{4}$ Division of Biostatistics, Mayo Clinic, Rochester, Minnesota.
}

Disclosure: Jeanne Huddleston discloses funding from the American Heart Association (\#03-30103N-04).

BACKGROUND: Hip operation (total hip arthroplasty [THA] or fracture repair) is the most common noncardiac surgical procedure performed in patients age 65 years and older.

OBJECTIVE: To determine the predictors of ischemic stroke in patients who have undergone hip operation.

DESIGN: Population-based historical cohort study, in which postoperative ischemic strokes were identified from medical record review for stroke diagnostic codes and brain imaging results and were confirmed by physician review.

SETTING: Tertiary care center in Olmsted County, Minnesota.

PATIENTS: Residents of Olmsted County who underwent hip surgical procedure.

MEASUREMENTS: Incidence of ischemic stroke within 1 year of hip operation.

RESULTS: In total, 1606 patients underwent 1886 hip procedures from 1988 through 2002 and were observed for ischemic stroke for 1 year after their procedure. Sixty-seven ischemic strokes were identified. The rate of stroke at 1 year after hip operation was $3.9 \%$. In univariate analysis, history of atrial fibrillation (hazard ratio [HR], 2.16; $P=0.005$ ), hip fracture repair vs. total hip arthroplasty (HR, 3.80; $P<0.001$ ), age 75 years or older (HR, 2.20; $P=0.02$ ), aspirin use (HR, $1.8 ; P=0.01$ ), and history of previous stroke (HR, 4.18; $P<0.001)$ were significantly associated with increased risk of stroke. In multivariable analysis, history of stroke (HR, 3.27; $P<0.001)$ and hip fracture repair (HR, 2.74; $P=0.004)$ were strong predictors of postoperative stroke.

CONCLUSIONS: This population-based historical cohort of patients with hip operation had a $3.9 \%$ cumulative probability of ischemic stroke over the first postoperative year. Hip fracture repair and history of stroke were the strongest predictors of this complication. Journal of Hospital Medicine 2009;4:298-303. ㅇ 2009 Society of Hospital Medicine.

KEYWORDS: arthroplasty, hip, hip fracture, ischemia, stroke.

In the United States, hip operations (internal fixation of fracture or total hip arthroplasty [THA]) are the most common noncardiac major surgical procedures performed in patients age 65 years and older (45.2 procedures per 100,000 persons per year). ${ }^{1}$ This number of procedures is projected to increase substantially in the coming decades.

Little is known about the clinical predictors of postoperative stroke in patients undergoing hip surgical procedures. Further, recent results of the Perioperative Ischemic Evaluation (POISE) trial have shown that measures taken to reduce cardiac complications postoperatively may adversely affect the risk of stroke. ${ }^{2}$ The POISE study showed decreases in myocardial infarction and coronary revascularization but accompanying increases in stroke and death with use of $\beta$-blockers in patients undergoing noncardiac surgery.

Prevention of adverse events is one of the top priorities of the U.S. health care system today. ${ }^{3-5}$ Risk stratification and therapeutic optimization of underlying chronic diseases may be important in decreasing perioperative risk and improving postoperative outcomes.
Our objective was to determine the rate of postoperative ischemic stroke in all residents of Olmsted County, MN, who underwent hip operation between 1988 and 2002 and to identify clinical predictors of postoperative stroke.

\section{Subjects and Methods}

Olmsted County is one of the few places in the world where comprehensive population-based studies of disease etiology and outcomes are feasible. This feasibility is due to the Rochester Epidemiology Project, a medical records linkage system that provides access to the records of all medical care in the community. ${ }^{1}$ All medical diagnoses made for a resident of Olmsted County are entered on a master sheet in the patient's medical record, which is then entered into a central computer index.

Hip operations were identified using the Surgical Information Recording System data warehouse, where detailed data are stored as International Classification of Diseases, 9th edition (ICD-9) codes for all surgical procedures performed from January 1, 1988, forward. A total of 2028 THAs 


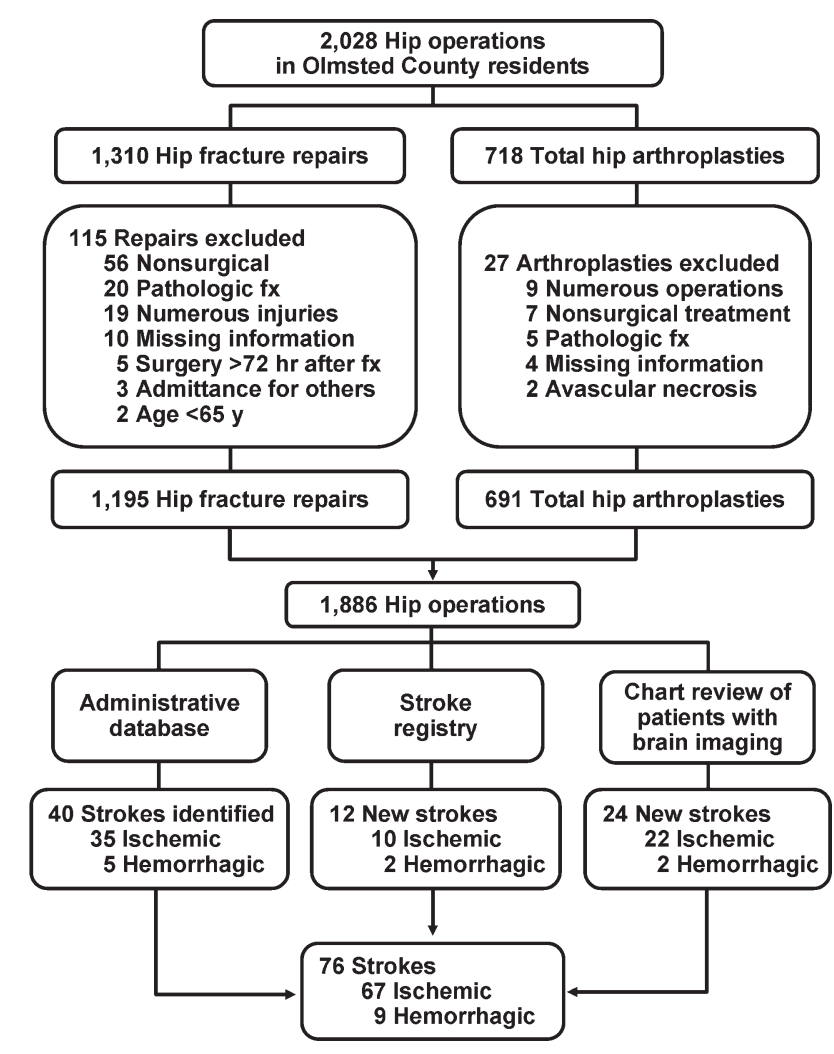

FIGURE 1. Flowchart showing subjects included in cohort of residents of Olmsted County, $\mathrm{MN}$, and methods of identification and types of strokes identified. Fx, fracture.

and hip fracture repairs (ICD-9 codes 81.51, 81.52, 81.53, 79.15, and 79.25) performed between 1988 and 2002 in Olmsted County were identified. Of the hip procedures, 142 were excluded (Figure 1). The final analysis cohort contained 1886 hip operations-1195 hip fracture repairs and 691 THAs.

The population-based cohort was assembled and the data were abstracted from complete inpatient and outpatient records from admission for surgical treatment up to 1 year after surgery. Only those patients who had given prior authorization for research were included in the study cohort. The Mayo Clinic Institutional Review Board approved the study.

\section{Case Ascertainment}

We used several screening procedures to completely enumerate all postoperative strokes in our study population (Figure 1). The Mayo Clinic administrative database was used to identify all cases with relevant cerebrovascular disease (ICD-9 codes 430.0-437.9, 368.12, 781.4, and 784.3) within 1 year after hip operation. The Rochester Stroke Registry identified incident cases of ischemic stroke in Olmsted County from 1988 through 1994. The clinic's administrative database was also used to identify brain imaging studies (brain computed tomography, magnetic res- onance imaging, or carotid ultrasonography) between the day of the procedure and 1 year postoperatively. A neurologist reviewed each image and the associated medical record identified during the screening process in detail for the constellation of signs and symptoms consistent with the diagnosis of stroke. Death certificates and autopsy reports were also reviewed to identify persons with the diagnosis of stroke. The outcome (stroke) was masked to the nurse abstractor who reviewed charts for predictors of postoperative stroke (eg, atrial fibrillation, coronary artery disease [CAD], history of stroke, medication use). The exposed or unexposed status of the patients to the predictors of stroke was masked to the physician (A.S.P.) who screened electronic medical records for the outcome measure (stroke).

"Cerebral infarction" or "ischemic stroke" was defined as the acute onset of a neurologic deficit that persisted for longer than 24 hours and corresponded to an arterial vascular territory of the cerebral hemispheres, brainstem, or cerebellum, with or without computed tomographic or magnetic resonance imaging documentation. "Transient ischemic attack" was defined as an episode of focal neurologic symptoms with abrupt onset and rapid resolution, lasting less than 24 hours, and due to altered circulation to a limited region of the brain.

Only patients with ischemic strokes clinically documented by a neurologist were included in the analysis.

\section{Primary Outcomes}

Outcomes were the cumulative probability of ischemic stroke and predictors of stroke in the first 12 months after surgical treatment of the hip.

\section{Statistical Analysis}

Continuous variables are presented as mean (standard deviation $[\mathrm{SD}]$ ); categorical variables are presented as number and percentage. Two-sample $t$ tests or Wilcoxon rank sum tests were used to test for differences between THAs and hip fracture repairs in demographic characteristics, past medical history, and baseline clinical data composed of continuous variables; $\chi^{2}$ or Fisher exact tests were used for categorical variables. No patient was lost to follow-up during the 1 year after the initial surgery. However, the data of patients who died or had a second hip procedure within that period were censored.

The rate of ischemic stroke within 1 year after the incident hip procedure was calculated using the Kaplan-Meier method. Second hip procedures within that period were counted as additional cases. Rates were calculated for the overall group, as well as for the univariate risk factors of operative procedure type, age, sex, past medical history of stroke, hypertension, atrial fibrillation, CAD, chronic obstructive pulmonary disease (COPD), diabetes mellitus, and chronic renal insufficiency. Use of $\beta$-blockers, hydroxymethylglutaryl-coenzyme A (HMG-CoA) reductase inhibitors, or aspirin at hospital admission was also considered. Cox 
proportional hazards regression models were used to evaluate the risk of ischemic stroke for each of these univariate risk factors. Multivariable Cox proportional hazards models were constructed with adjustments for operative procedure type, age, sex, and comorbid conditions such as atrial fibrillation and hypertension. These covariates were added in a stepwise selection to identify factors significantly associated with the outcome. To account for patients who had a second hip procedure within 1 year of their first operation, we calculated all Cox proportional hazards regression results using the robust sandwich estimate of the covariance matrix. The proportional hazards assumption for all Cox models was evaluated with the methods proposed by Therneau and Grambsch; ${ }^{6}$ no violations of this assumption were identified. The rate of postoperative stroke after adjusting for the competing risk of death was calculated using the approach of Gooley et al. ${ }^{7}$ All statistical tests were 2-sided, and a $P$ value was considered significant if it was less than 0.05 . Statistical analyses were performed using statistical software (SAS version 9.1.3; SAS Institute, Inc., Cary, NC).

\section{Results}

Among the patients with the 1886 hip procedures, 67 ischemic strokes were identified within 1 year after the index surgical procedure-10 (1.4\%) among the 691 THAs and 57 $(4.8 \%)$ among the 1195 hip fracture repairs. Baseline characteristics are summarized in Table 1. Compared with the THA group, patients in the hip fracture repair group were more likely to be older and female. Additionally, such comorbid conditions as a history of stroke, diabetes mellitus, congestive heart failure, atrial fibrillation, or dementia were more prevalent in the hip fracture repair group.

Univariate analyses assessing the rate and risk of postoperative ischemic stroke are shown in Table 2. The rate of stroke was significantly greater among hip fracture repairs than THAs 30 days postoperatively and 1 year postoperatively $(1.5 \%$ vs. $0.6 \%$ and $5.5 \%$ vs. $1.5 \%$, respectively; $P<$ 0.001 ) (Figure 2). In our study we found an annual incidence rate of ischemic stroke of 4093 per 100,000 personyears (95\% confidence interval [CI], 3172-5198 per 100,000 person-years). Accounting for death as a competing risk for stroke had little impact on the rate of stroke overall or within the 2 surgical groups (results not shown). Univariate Cox proportional hazards models showed that neither sex nor history of hypertension, diabetes mellitus, COPD, chronic renal insufficiency, or CAD or use of HMG-CoA reductase inhibitors or $\beta$-blockers were significant predictors of ischemic stroke. However, other clinical risk factors, such as a history of atrial fibrillation (hazard ratio [HR], 2.16; $P=$ 0.005), hip fracture repair vs. THA (HR, 3.80; $P<0.001$ ), increased age (HR, 2.20; $P=0.017$ ), aspirin use (HR, 1.8; $P$ $=0.014)$, and history of previous stroke (HR, 4.18; $P<$ 0.001 ), were significantly associated with an increased risk of stroke (Table 2).

\begin{tabular}{|c|c|c|c|c|}
\hline \multirow[b]{2}{*}{ Characteristics } & \multicolumn{2}{|c|}{ Surgical Procedure } & \multirow[b]{2}{*}{$\begin{array}{l}\text { Total } \\
(\mathrm{n}=1,886)\end{array}$} & \multirow[b]{2}{*}{$P$ Value* } \\
\hline & $\begin{array}{l}\text { THA } \\
(\mathrm{n}=691)\end{array}$ & $\begin{array}{l}\text { Fracture Repair } \\
(\mathrm{n}=1,195)\end{array}$ & & \\
\hline Age, years & $74.9(6.59)$ & $84.2(7.49)$ & $80.8(8.46)$ & $<0.001$ \\
\hline Sex, male & 258 (37.3) & 234 (19.6) & 492 (26.1) & $<0.001$ \\
\hline Race, White & $690(100)$ & 1,187 (99.3) & 1,877 (99.5) & 0.17 \\
\hline $\mathrm{BMI}^{\dagger}$ & $27.7(5.36)$ & $23.3(4.93)$ & $24.9(5.52)$ & $<0.001$ \\
\hline \multicolumn{5}{|l|}{ History } \\
\hline Hypertension & $424(61.4)$ & 695 (58.2) & 1,119 (59.3) & 0.17 \\
\hline Diabetes & $57(8.2)$ & 141 (11.8) & 198 (10.5) & 0.02 \\
\hline Stroke & $50(7.2)$ & $334(27.9)$ & $384(20.4)$ & $<0.001$ \\
\hline CHF & $100(14.5)$ & $321(26.9)$ & $421(22.3)$ & $<0.001$ \\
\hline Atrial fibrillation & $72(10.4)$ & $241(20.2)$ & $313(16.6)$ & $<0.001$ \\
\hline Dementia & $16(2.3)$ & $407(34.1)$ & $423(22.4)$ & $<0.001$ \\
\hline ASA risk classification ${ }^{\ddagger}$ & & & & $<0.001$ \\
\hline 1 or 2 & $343(49.6)$ & $172(14.4)$ & 515 (27.3) & \\
\hline 3,4, or 5 & $348(50.4)$ & $1,022(85.6)$ & $1,370(72.7)$ & \\
\hline \multicolumn{5}{|l|}{ Medication on admission } \\
\hline Aspirin & $168(24.3)$ & $369(30.9)$ & $537(28.5)$ & 0.002 \\
\hline$\beta$-Blocker & $134(19.4)$ & $184(15.4)$ & $318(16.9)$ & 0.03 \\
\hline Insulin & $12(1.7)$ & $48(4)$ & $60(3.2)$ & 0.007 \\
\hline Length of stay, days & $7.3(3.9)$ & $10.0(7.61)$ & $9.0(6.63)$ & $<0.001$ \\
\hline \multicolumn{5}{|c|}{$\begin{array}{l}\text { NOTE: Continuous variables are represented as mean (SD); categorical variables are represented as } \\
\text { number and percentage. }\end{array}$} \\
\hline \multicolumn{5}{|c|}{$\begin{array}{l}\text { Abbreviations: ASA, American Society of Anesthesiologists; BMI, body mass index; CHF, congestive } \\
\text { heart failure; THA, total hip arthroplasty. }\end{array}$} \\
\hline \multirow{2}{*}{\multicolumn{5}{|c|}{$\begin{array}{l}{ }^{*} P \text { values are from Kruskal-Wallis tests for continuous variables and from either } \chi^{2} \text { or Fisher exact tests } \\
\text { for categorical variables. }\end{array}$}} \\
\hline \multicolumn{2}{|c|}{ 'Fifteen cases had no BMI data. } & & & \\
\hline${ }^{\ddagger}$ One case had no ASA risk c & fication data. & & & \\
\hline
\end{tabular}

Because age was associated with the type of surgical procedure $(87 \%$ of hip fracture repair patients were 75 years or older compared with $45 \%$ of THA patients), the effect of hip fracture repair on ischemic stroke was adjusted for age. For similar reasons, sex was also examined as an adjusting factor. Adjustment for age and sex resulted in only a slight attenuation of the HR for hip fracture repair vs. THA, from 3.8 to 3.4. A further analysis also adjusted for history of hypertension and history of atrial fibrillation, both comorbidities commonly associated with ischemic stroke. After adjustment for age, sex, history of hypertension, and history of atrial fibrillation, the risk of ischemic stroke was still significantly greater in the hip fracture repair group than in the THA group (HR, 2.8; 95\% CI, 1.4-5.7; $P=0.005$ ).

To determine the most important predictors of postoperative ischemic stroke, multivariable analysis was conducted with stepwise selection. Potential risk factors included the following: operative procedure type (hip fracture repair vs. THA), age, sex, and history of stroke, hypertension, atrial fibrillation, CAD, COPD, diabetes mellitus, and chronic renal insufficiency, as well as use of $\beta$-blockers, HMG-CoA reductase inhibitors, and aspirin on hospital admission. Among all these factors, history of stroke (HR, 3.27; $P<0.001)$ and hip fracture repair vs. THA (HR, 2.74; $P=0.004)$ were 


\section{TABLE 2. Univariate Estimates and Predictors of Postoperative Ischemic Stroke After Hip Operation}

\begin{tabular}{|c|c|c|c|c|c|c|}
\hline Variable & Number of Patients & Number of Events & \multicolumn{2}{|c|}{ Rate $(\%)$} & Hazard Ratio & $P$ Value \\
\hline Overall & 1886 & 67 & $1.2(0.7-1.7)$ & $3.9(3-4.8)$ & & \\
\hline \multicolumn{7}{|l|}{$\begin{array}{l}\text { Type of operative } \\
\text { procedure }\end{array}$} \\
\hline THA & 691 & 10 & $0.6(0.0-1.1)$ & $1.5(0.6-2.4)$ & & \\
\hline Hip fracture repair & 1195 & 57 & $1.5(0.8-2.2)$ & $5.5(4.1-6.9)$ & 3.80 (1.94-7.44) & $<0.001$ \\
\hline$\geq 75$ & 1358 & 56 & $1.3(0.7-1.9)$ & $4.7(3.5-5.8)$ & $2.20(1.15-4.21)$ & 0.02 \\
\hline \multicolumn{7}{|l|}{ Sex } \\
\hline Female & 1394 & 54 & $1.3(0.7-1.9)$ & $4.2(3.1-5.3)$ & & \\
\hline Male & 492 & 13 & $0.8(0.0-1.7)$ & $2.9(1.3-4.4)$ & 0.69 (0.38-1.27) & 0.24 \\
\hline \multicolumn{7}{|l|}{ History of stroke } \\
\hline No & 1502 & 34 & $0.7(0.3-1.2)$ & $2.4(1.6-3.3)$ & & \\
\hline \multicolumn{7}{|l|}{$\begin{array}{l}\text { History of atrial } \\
\text { fibrillation }\end{array}$} \\
\hline No & 1573 & 48 & $1.0(0.5-1.5)$ & $3.3(2.4-4.2)$ & & \\
\hline Yes & 313 & 19 & $1.9(0.4-3.5)$ & $7.0(3.9-9.9)$ & $2.16(1.27-3.67)$ & 0.005 \\
\hline \multicolumn{7}{|l|}{ History of CAD } \\
\hline No & 1224 & 40 & $1.1(0.5-1.6)$ & $3.5(2.4-4.5)$ & & \\
\hline Yes & 662 & 27 & $1.4(0.5-2.3)$ & $4.7(2.9-6.4)$ & $1.34(0.82-2.19)$ & 0.24 \\
\hline \multicolumn{7}{|l|}{ History of COPD } \\
\hline No & 1606 & 62 & $1.4(0.8-2.0)$ & $4.2(3.1-5.2)$ & & \\
\hline Yes & 280 & 5 & $0(0.0-0.0)$ & $2.2(0.3-4.1)$ & $0.49(0.20-1.22)$ & 0.13 \\
\hline \multicolumn{7}{|l|}{$\begin{array}{l}\text { History of diabetes } \\
\text { mellitus }\end{array}$} \\
\hline No & 1688 & 56 & $1.1(0.6-1.7)$ & $3.6(2.7-4.5)$ & & \\
\hline \multicolumn{7}{|l|}{$\beta$-Blocker use } \\
\hline No & 1568 & 52 & $1.1(0.6-1.6)$ & $3.6(2.7-4.6)$ & & \\
\hline Yes & 318 & 15 & $1.6(0.2-3.0)$ & $5.1(2.6-7.6)$ & $1.42(0.81-2.52)$ & 0.22 \\
\hline \multicolumn{7}{|l|}{$\begin{array}{l}\text { HMG-CoA reductase } \\
\text { inhibitor use }\end{array}$} \\
\hline No & 1736 & 63 & $1.2(0.7-1.7)$ & $4.0(3.0-4.9)$ & & \\
\hline $\begin{array}{l}\text { Yes (statin/other lipid } \\
\text { lowering drugs) }\end{array}$ & 148 & 4 & $1.4(0-3.2)$ & $2.8(0.1-5.4)$ & $0.70(0.26-1.94)$ & 0.50 \\
\hline
\end{tabular}

Abbreviations: CAD, coronary artery disease; CI, confidence interval; COPD, chronic obstructive pulmonary disease; HMG-CoA, hydroxymethyglutaryl coenzyme A; THA, total hip arthroplasty.

confirmed to be significant predictors of postoperative ischemic stroke; the other factors did not significantly affect the model (Figure 2).

\section{Comment}

Our findings contrast those of previous studies that focused on perioperative ischemic stroke rates for specific surgical procedures, ${ }^{2,8,9}$ but do seem concordant with published results for early event rates of cerebrovascular accident or transient ischemic attack (1\%) following hip fracture. ${ }^{10}$ The data from our study suggest that perioperative stroke cumulative probability is relatively high for hip procedures at both 30 days $(1.2 \%)$ and 1 year $(3.9 \%)$ after the index surgical procedure compared with general procedures. Subjects with a history of stroke who were undergoing hip operation 


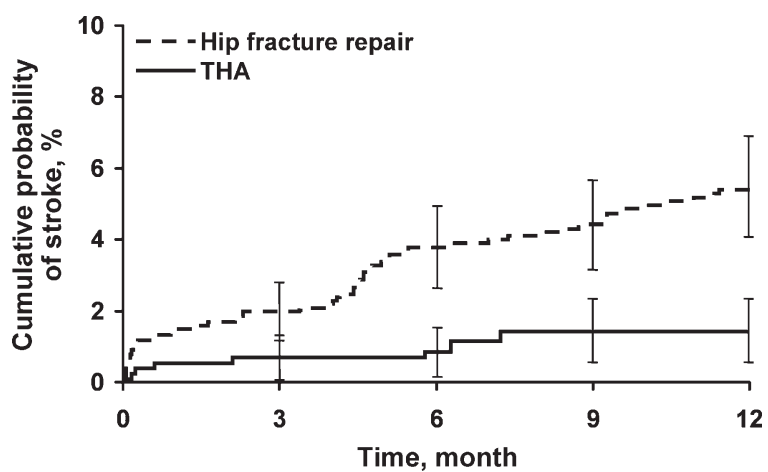

FIGURE 2. Kaplan-Meier curves of cumulative probability of ischemic stroke after hip fracture repair vs. total hip arthroplasty (THA). Error bars indicate 95\% confidence intervals; $P<0.001$; hazard ratio $=3.8$.

had a postoperative stroke risk of $3.0 \%$ at 30 days and $9.9 \%$ at 1 year.

The incidence of stroke was greater in the hip fracture repair group ( $1.5 \%$ at 30 days and $5.5 \%$ at 1 year) than in the elective THA group $(0.6 \%$ at 30 days and $1.5 \%$ at 1 year). The increased 1-year mortality for patients undergoing hip surgery compared with the general population is in part due to cerebrovascular disease, ${ }^{10}$ and, therefore, the 1 -year stroke incidence is important.

After adjustment for age, sex, and comorbidities (hypertension and atrial fibrillation), the risk of postoperative ischemic stroke was 2.71 times greater in the hip fracture repair group than in the THA group $(P=0.006)$. These data are important in counseling and caring for patients undergoing different types of hip procedures.

From 1985 through 1989, for the age group (75-84 years old) that best fits the demographics of our cohort, both men and women had limited variation over time in annual incidence rates of stroke (2149-1074 strokes per 100,000 population per year) for Olmsted County, MN. ${ }^{11}$ In our study we found an annual incidence rate of ischemic stroke of 4,093 per 100,000 person-years (95\% CI, 3172-5198 per 100,000 person-years). The lower limit of the $95 \% \mathrm{CI}$ is higher than the rates reported for Olmsted County, suggesting that having hip surgery increases the 1-year risk of ischemic stroke.

Previous studies have shown that the risk factor most consistently correlated to perioperative ischemic stroke is a history of stroke. ${ }^{9}$ In our study, history of stroke and type of hip fracture surgery were confirmed to be the strongest predictors of postoperative stroke. History of hypertension, atrial fibrillation, CAD, COPD, diabetes, or chronic renal insufficiency was not correlated to perioperative ischemic stroke.

Nonmodifiable risk factors, such as advanced age, serve as markers of stroke risk and help identify high-risk populations that may require aggressive intervention. After age adjustment of hip fracture repair, age was no longer significantly associated with postoperative stroke.
Cerebrovascular disease appears to be a marker for CAD, and, therefore, patients with a history of stroke usually have a Revised Cardiac Risk Index that may suggest the use of $\beta$ blockers. According to the recent results of the POISE trial, use of $\beta$-blockers could lead to increased stroke incidence. ${ }^{2}$ Our results showed no significant correlation between stroke risk and $\beta$-blocker use, but our study period was from 1988 to 2002, when titration of $\beta$-blocker dose to heart rates of 55 to 60 beats per minute was not common practice.

Several studies have confirmed the value of aspirin in decreasing the rate of vascular outcomes after diagnosis of transient ischemic attack or stroke. ${ }^{12}$ In our study, aspirin use on hospital admission was found in the univariate analysis to be associated with an increased risk of stroke, but this finding was not confirmed after adjustments for age, sex, and comorbid conditions. Aspirin use on admission was not a significant predictor of postoperative stroke, most likely because aspirin use can be considered a marker of increased cardiovascular risk and we adjusted for these comorbid conditions.

The limitations of this study are inherent in its retrospective design. First, we identified all incident cases of stroke after hip operation by reviewing medical records and then abstracting data from those records. We may have missed some mild strokes if they were misclassified as peripheral vestibular neuropathy, migraine, or even seizure. Less likely is that we missed strokes within the first 30 days after the procedure because that is the period in which patients with hip operation are either hospitalized or sent for rehabilitation in skilled nursing facilities. It is known that institutionalization leads to better surveillance and more complete ascertainment of any medical event.

The event rate of postoperative stroke at 30 days after hip operation was low. Therefore, we did not have the statistical power to comment meaningfully on predictors of stroke at 30 days after the hip procedure. Any nonrespondent or volunteer bias was addressed by using data from the Rochester Epidemiology Project, which allowed us to identify all Olmsted County residents who underwent hip operation between 1988 and 2002. The diagnostic suspicion bias was also accounted for in our study design because different physicians provided care and outcome measurement.

Our results apply for the patients who underwent hip operation between 1988 and 2002. The noncardiac surgery guidelines have been revised between 1988 and 2002, and we did not perform a stratified analysis by index year. The next step in our study will be to extend our data collection to 2008 and look at time trends.

\section{Conclusion}

In this population-based historical cohort study, patients undergoing hip operation had a $3.9 \%$ cumulative probability of ischemic stroke during the first postoperative year. History of stroke and type of hip procedure (ie, hip fracture repair) were the strongest predictors of this complication. Because 
history of stroke is such a strong predictor of postoperative stroke, the perioperative management of these patients should probably be tailored, with closely observed blood pressure management and antihypertensive medication adjustment, to avoid compromising cerebral perfusion. Also, to avoid postoperative hypercoagulability that increases the risk of stroke, these patients may need to begin receiving antiplatelets as soon as is surgically acceptable. ${ }^{13-15}$

\section{Address for correspondence and reprint requests:}

Jeanne M. Huddleston, MD, Division of Hospital Internal Medicine, Mayo Clinic, 200 First Street SW, Rochester, MN 55905;

Telephone: 507-284-8917; Fax: 507-284-2107;

E-mail: huddleston.jeanne@mayo.edu Received 28 October 2008; revision received 9 March 2009; accepted 21 March 2009.

\section{References}

1. Melton LJ 3rd. History of the Rochester Epidemiology Project. Mayo Clin Proc. 1996;71(3):266-274.

2. POISE Study Group; Devereaux PJ, Yang H, Yusuf S, Guyatt G, Leslie K, Villar JC, et al. Effects of extended-release metoprolol succinate in patients undergoing non-cardiac surgery (POISE trial): a randomised controlled trial. Lancet. 2008;371(9627):1839-1847.

3. Thom T, Haase N, Rosamond W, Howard VJ, Rumsfeld J, Manolio T, et al; American Heart Association Statistics Committee and Stroke Statistics Subcommittee. Heart disease and stroke statistics-2006 update: a report from the American Heart Association Statistics Committee and Stroke Statistics Subcommittee. Circulation. 2006;113(6):e85-e151. [Errata in: Circulation. 2006;113(14):e696 and Circulation. 2006;114(23):e630.
4. Shojania KG, Duncan BW, McDonald KM, Wachter RM, Markowitz AJ, eds. Making health care safer: a critical analysis of patient safety practices. Evidence Report/Technology Assessment No. 43. AHRQ publication no. 01-E058. Rockville, MD: Agency for Healthcare Research and Quality (AHRQ), U.S. Department of Health and Human Services; 2001. 668 p.

5. McDonald CJ, Weiner M, Hui SL. Deaths due to medical errors are exaggerated in Institute of Medicine report. JAMA. 2000;284(1):93-95.

6. Therneau TM, Grambsch PM. Modeling survival data: extending the Cox model. New York: Springer; 2000.

7. Gooley TA, Leisenring W, Crowley J, Storer BE. Estimation of failure probabilities in the presence of competing risks: new representations of old estimators. Stat Med. 1999;18(6):695-706.

8. Larsen SF, Zaric D, Boysen G. Postoperative cerebrovascular accidents in general surgery. Acta Anaesthesiol Scand. 1988;32(8):698-701.

9. Landercasper J, Merz BJ, Cogbill TH, Strutt PJ, Cochrane RH, Olson RA, et al. Perioperative stroke risk in 173 consecutive patients with a past history of stroke. Arch Surg. 1990;125(8):986-989.

10. Lawrence VA, Hilsenbeck SG, Noveck H, Poses RM, Carson JL. Medical complications and outcomes after hip fracture repair. Arch Intern Med. 2002;162(18):2053-2057.

11. Brown RD, Whisnant JP, Sicks JD, O'Fallon WM, Wiebers DO. Stroke incidence, prevalence, and survival: secular trends in Rochester, Minnesota, through 1989. Stroke. 1996;27(3):373-380.

12. CAST (Chinese Acute Stroke Trial) Collaborative Group. Randomised placebo-controlled trial of early aspirin use in 20,000 patients with acute ischaemic stroke. Lancet. 1997;349(9066):1641-1649.

13. Dixon B, Santamaria J, Campbell D. Coagulation activation and organ dysfunction following cardiac surgery. Chest. 2005;128(1):229-236.

14. Páramo JA, Rifón J, Llorens R, Casares J, Paloma MJ, Rocha E. Intra- and postoperative fibrinolysis in patients undergoing cardiopulmonary bypass surgery. Haemostasis. 1991;21(1):58-64.

15. Selim M. Perioperative stroke. N Engl J Med. 2007;356(7):706-713. 